\title{
Métodos de soma térmica e datas de semeadura na determinação de filocrono de cultivares de trigo
}

\author{
Hamilton Telles Rosa(1), Lidiane Cristine Walter ${ }^{(1)}$, Nereu Augusto Streck ${ }^{(1)}$ e Cleber Maus Alberto ${ }^{(2)}$ \\ (1)Universidade Federal de Santa Maria, Departamento de Fitotecnia, Avenida Roraima, № 1.000, Bairro Camobi, CEP 97105-900 Santa \\ Maria, RS. E-mail: a1000tontr@gmail.com, lidianewalter@gmail.com, nstreck2@yahoo.com.br (2)Universidade Federal do Pampa, Rua Luiz \\ Joaquim de Sá Sabrito, s/no, Bairro Promorar, CEP 97650-000 Itaqui, RS. E-mail: cleberalb@yahoo.com.br
}

Resumo - O objetivo deste trabalho foi avaliar o efeito dos métodos de cálculo de graus-dia e de datas de semeadura na determinação do filocrono de cultivares de trigo (Triticum aestivum). Foi conduzido um experimento em campo, com 13 datas de semeadura, durante três anos (2005-2007), em Santa Maria, RS, usando-se seis cultivares de trigo: BRS Louro, CEP 52, CEP 51, BRS 177, Nova Era e BRS Tarumã. O filocrono $\left({ }^{\circ} \mathrm{C}\right.$ dia folha $\left.{ }^{-1}\right)$ foi estimado pelo inverso do coeficiente angular da regressão linear entre o estádio de Haun e a soma térmica acumulada calculada por seis métodos. O método de cálculo da soma térmica afeta as estimativas do filocrono em trigo.O uso das três temperaturas cardinais de emissão de folhas e a comparação das temperaturas cardinais com a temperatura mínima e máxima do ar melhora a precisão na simulação do desenvolvimento vegetativo dessa cultura, em comparação aos demais métodos. O filocrono varia com a cultivar de trigo e com a data de semeadura, e cultivares mais precoces (BRS Louro e CEP 51) têm menor filocrono do que cultivares tardias (Nova Era e BRS Tarumã). Em semeaduras no outono ou inverno, o filocrono é maior do que em semeaduras de primavera ou verão.

Termos para indexação: Triticum aestivum, aparecimento de folhas, desenvolvimento vegetal, fotoperíodo, temperatura.

\section{Thermal time methods and sowing dates in phyllochron determination in wheat cultivars}

\begin{abstract}
The objective of this work was to evaluate the effect of degree-days and sowing dates calculation methods on phyllochron determination in wheat (Triticum aestivum) cultivars. A three-year field experiment with 13 sowing dates was conducted for three years (2005-2007) in Santa Maria, RS, Brazil, using six wheat cultivars: BRS Louro, CEP 52, CEP 51, BRS 177, Nova Era and BRS Tarumã. The phyllochron $\left({ }^{\circ} \mathrm{C}\right.$ day leaf $\left.{ }^{-1}\right)$ was estimated by the inverse of the slope of the linear regression of the Haun stage against the accumulated thermal time calculated using six methods. The thermal time calculation method affects wheat phyllochron estimates. The use of the three cardinal temperatures for leaf emission and cardinal temperatures compared to the minimum and maximum air temperatures improves the precision of the vegetative development simulation of this crop, in comparison to the other methods. The phyllochron varies with wheat cultivar and sowing date, and early cultivars (BRS Louro and CEP 51) have lower phyllochron than late cultivars (Nova Era and BRS Tarumã). The phyllochron is greater in sowings carried out during fall and winter than in the ones carried out during spring and summer.
\end{abstract}

Index terms: Triticum aestivum, leaf appearance, plant development, photoperiod, temperature.

\section{Introdução}

O trigo (Triticum aestivum L.) é a segunda cultura de grãos mais produzida no mundo (United States Department of Agriculture, 2009) e a principal cultura de inverno no Brasil. Novas cultivares são lançadas no mercado a cada ano, e caracterizar o crescimento e o desenvolvimento desses genótipos é importante para avaliar o seu desempenho agronômico diante das variações da temperatura do ar.
Um indicador de desenvolvimento vegetal bastante usado em estudos que envolvem datas de semeadura é o número de folhas acumuladas (NF) na haste principal (Streck et al., 2003b, 2006; Paula et al., 2005). O NF está relacionado com a evolução da área foliar que influencia diretamente a interceptação da radiação solar (Sinclair et al., 2004; Dellai et al., 2005). Diversos estádios do ciclo de desenvolvimento do trigo também estão associados com o NF, como o início e o fim da diferenciação da espiga, a emissão de afilhos, o emborrachamento e o aparecimento da espiga (McMaster et al., 1991; Streck

Pesq. agropec. bras., Brasília, v.44, n.11, p.1374-1382, nov. 2009 
et al., 2003a,b). Em razão disso, o NF é importante em diversos modelos de simulação de culturas agrícolas, e é obtido integrando-se a velocidade de emissão de folhas (VEF) em relação ao tempo (McMaster et al., 1991; Streck et al., 2003a; Paula et al., 2005). Uma maneira de estimar a VEF é por meio do conceito de filocrono, que é o intervalo de tempo entre a emissão de duas folhas sucessivas em um colmo, expresso em unidade de tempo por folha (Frank \& Bauer, 1995; Hermes et al., 2001). O filocrono depende da cultivar, da altura do colmo e do tempo para o início da elongação da folha (Skinner \& Nelson, 1995). Fatores ambientais, como temperaturas extremas, estresses hídrico e nutricional e o fotoperíodo, também afetam o filocrono (Wilhelm \& McMaster, 1995). Além desses, a data de semeadura é outro fator com efeito sobre o filocrono em trigo, já que os filocronos das semeaduras de outono e inverno são maiores do que os das semeaduras fora dessas épocas (Jamieson et al., 2008; Bassu et al., 2009).

$\mathrm{Na}$ quantificação do filocrono, o tempo pode ser expresso em dias, porém as plantas têm seu crescimento e desenvolvimento fortemente influenciados por variáveis ecológicas, principalmente a temperatura do ar (McMaster, 2005). Assim, deve-se incluir a temperatura na contabilização do tempo vegetal, e a maneira clássica de se fazer essa inclusão é pelo uso da soma térmica, cuja unidade é o grau dia (Gilmore Junior \& Rogers, 1958), definido como a soma diária de unidades térmicas acima de uma temperatura base inferior, abaixo da qual a planta não se desenvolve ou seu desenvolvimento é tão lento que pode ser desprezado (McMaster \& Wilhelm, 1997). Assim, o filocrono tem como unidade $\mathrm{o}^{\circ} \mathrm{C}$ dia folha ${ }^{-1}$.

Existem vários métodos de cálculo da soma térmica, e eles podem ser agrupados em duas categorias. Uma está relacionada ao uso ou não das três temperaturas cardinais ( $\mathrm{Tb}$, temperatura base inferior; Tot, temperatura ótima; e TB, temperatura base superior): na equação mais simples da soma térmica, subtrai-se a temperatura média diária do ar da Tb (Gilmore Junior \& Rogers, 1958), mas também pode-se considerar um valor máximo de acúmulo térmico diário na Tot; para temperaturas supraótimas, em que o acúmulo térmico não é maior do que o acúmulo na Tot, pode-se até mesmo considerar um decréscimo linear até zero do acúmulo térmico na TB (Streck et al., 2007b). Outro enfoque refere-se ao momento de se comparar a temperatura do ar com as temperaturas cardinais de desenvolvimento: a comparação pode ser feita com a temperatura mínima e máxima do ar, ou então, calcula-se a temperatura média do ar ( $\left.\mathrm{T}_{\text {méd }}\right)$ e compara-se a $\mathrm{T}_{\text {méd }}$ com a Tb, Tot ou TB (McMaster \& Wilhelm, 1997). Os métodos, nos dois enfoques, podem resultar em valores de soma térmica distintos para um mesmo período, especialmente em épocas do ano com temperaturas abaixo ou acima das temperaturas cardinais de desenvolvimento da cultura (McMaster \& Wilhelm, 1997). Consequentemente, valores diferentes de filocrono podem ser obtidos de acordo com o método de cálculo da soma térmica usado, o que resulta em insegurança na análise da variação do filocrono entre cultivares, entre datas de semeadura e entre anos (Streck et al., 2007a). Apesar dos inúmeros trabalhos em que o filocrono foi determinado na cultura do trigo (Frank \& Bauer, 1995; Xue et al., 2004), inclusive no Brasil (Streck et al., 2005b), é pertinente estender a análise do filocrono considerando diferentes métodos de cálculo da soma térmica em diferentes datas de semeadura, já que esse enfoque não foi considerado nos trabalhos anteriores.

O objetivo deste trabalho foi avaliar o efeito dos métodos de cálculo de graus-dia e de data de semeadura na determinação do filocrono de cultivares de trigo.

\section{Material e Métodos}

Um experimento de campo foi realizado na área experimental do Departamento de Fitotecnia da Universidade Federal de Santa Maria, Santa Maria,

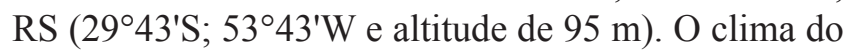
local, segundo a classificação climática de Köppen, é Cfa, subtropical úmido sem estação seca definida e com verões quentes.

O experimento foi conduzido durante três anos (2005-2007). Durante esse período, foram usadas 13 datas de semeadura, espaçadas a intervalos de aproximadamente 45 dias: $24 / 6,4 / 8,7 / 9,26 / 10$ e $21 / 12$ em 2005 , e $6 / 2,21 / 3,12 / 5,7 / 7,4 / 9,25 / 10$ em 2006, 18/4 e 7/6 em 2007. Essas datas situam-se antes, durante e depois da época recomendada para a cultura do trigo no local, que é de 11 de maio a 20 de julho (Reunião da Comissão Sul-Brasileira de Pesquisa de Trigo e Triticale, 2006). Essa ampla faixa de datas de semeadura é importante neste tipo de estudo, para que as plantas fiquem expostas a diferentes condições meteorológicas durante seu desenvolvimento. Foram utilizadas seis cultivares de trigo: CEP 51, CEP 52 e Nova Era, da Fundação Centro de Experimentação e Pesquisa (Fundacep), 
e BRS Louro, BRS 177 e BRS Tarumã, da Empresa Brasileira de Pesquisa Agropecuária. Essas cultivares foram utilizadas por serem indicadas para o Rio Grande do Sul e por apresentarem ciclos de desenvolvimento diferentes: precoce (CEP 51 e BRS Louro), médio (CEP 52 e BRS 177) e tardio (Nova Era e BRS Tarumã) (Reunião da Comissão Sul-Brasileira de Pesquisa de Trigo e Triticale, 2006). A cultivar BRS Tarumã é classificada como trigo de duplo propósito, ou seja, serve para pastagem e produção de grãos.

O delineamento experimental foi o de blocos ao acaso, em arranjo fatorial $6 \times 13$, com seis cultivares e 13 épocas de semeadura, e quatro repetições. O solo foi preparado pelo método convencional, com duas gradagens. A adubação foi realizada de acordo com a análise química do solo, seguindo as recomendações de adubação e calagem para os estados do Rio Grande do Sul e Santa Catarina (Sociedade Brasileira de Ciência do Solo, 2004). A densidade de semeadura foi de 330 sementes por metro quadrado. Cada repetição foi composta por uma parcela de quatro linhas espaçadas em $0,20 \mathrm{~m}$ e com 2,40 m de comprimento. Os tratos culturais, como controle de insetos, doenças e plantas invasoras, foram realizados sempre que necessário, de forma a evitar o estresse por estes fatores. Não houve restrição hídrica para as plantas durante o ciclo de desenvolvimento, uma vez que a precipitação pluvial foi elevada. Apenas nas datas de semeadura de $21 / 12 / 2005$ e $6 / 2 / 2006$, as parcelas foram irrigadas por aspersão para manter a água no solo acima de $50 \%$ da fração de água disponível no solo, já que nessas duas épocas a demanda evaporativa do ar foi elevada em razão da alta temperatura do ar, do fotoperíodo longo (acima de 14 horas) e dos altos valores de radiação solar, característicos do verão nesse local.

A emergência foi considerada quando $50 \%$ das plantas em 0,5 $\mathrm{m}$ de cada linha da parcela estavam visíveis acima do nível do solo (Streck et al., 2003a,b). As datas de emergência observadas nas 13 épocas de semeadura citadas anteriormente foram, respectivamente: $30 / 6,14 / 8,16 / 9,1 / 11,27 / 12$ em 2005, 14/2, 28/3, 24/5, 18/7, 13/9, 3/11 em 2006, 25/4 e 14/6 em 2007. Uma semana após a emergência, seis plantas de trigo foram selecionadas aleatoriamente nas linhas centrais de cada parcela e identificadas com arames coloridos. Uma vez por semana, foi medido, no colmo principal dessas plantas, o comprimento da última e da penúltima folha, e foi contado o número de folhas até o aparecimento da última folha (folha bandeira). Com esses dados, foi calculado o estádio de Haun (HS, folhas) no colmo principal, que representa o número de folhas completamente expandidas (NF) mais a razão entre o comprimento da última $\left(\mathrm{L}_{\mathrm{n}}\right)$ e o comprimento da penúltima $\left(\mathrm{L}_{\mathrm{n}-1}\right)$ folha, por meio da equação (Streck et al., 2005b):

$\mathrm{HS}=(\mathrm{NF}-1)+\mathrm{L}_{\mathrm{n}} / \mathrm{L}_{\mathrm{n}-1}$.

As temperaturas mínima e máxima diárias do ar, correspondentes ao período experimental, foram obtidas na estação meteorológica convencional, pertencente ao Oitavo Distrito de Meteorologia do Instituto Nacional de Meteorologia, localizada a aproximadamente a $80 \mathrm{~m}$ da área experimental.

A soma térmica diária $\left(\mathrm{STd},{ }^{\circ} \mathrm{C}\right.$ dia $)$ foi calculada pelos métodos (Gilmore Junior \& Rogers, 1958; Streck et al., 2007a,b):

Método 1.1: $\mathrm{STd}=\left(\mathrm{T}_{\text {méd }}-\mathrm{Tb}\right) .1$ dia, se $\mathrm{T}_{\text {méd }}<\mathrm{Tb}$, então $\mathrm{T}_{\text {méd }}=\mathrm{Tb}$.

Método 1.2: $\mathrm{STd}=\left(\mathrm{T}_{\text {méd }}-\mathrm{Tb}\right) .1$ dia, se $\mathrm{T}_{\text {min }} \leq \mathrm{Tb}$, então $\mathrm{T}_{\text {min }}=\mathrm{Tb}$.

Método 2.1: $\mathrm{STd}=\left(\mathrm{T}_{\text {méd }}-\mathrm{Tb}\right) .1$ dia, se $\mathrm{T}_{\text {méd }}<\mathrm{Tb}$, então $\mathrm{T}_{\text {méd }}=\mathrm{Tb}$; se $\mathrm{T}_{\text {méd }}>$ Tot, então $\mathrm{T}_{\text {méd }}=$ Tot.

Método 2.2: $\mathrm{STd}=\left(\mathrm{T}_{\text {méd }}-\mathrm{Tb}\right) .1$ dia, se $\mathrm{T}_{\min }<\mathrm{Tb}$, então $\mathrm{T}_{\text {min }}=\mathrm{Tb}$ e, se $\mathrm{T}_{\text {máx }}>$ Tot, então $\mathrm{T}_{\text {máx }}=$ Tot.

Método 3.1: $\mathrm{STd}=\left(\mathrm{T}_{\text {méd }}-\mathrm{Tb}\right) .1$ dia, quando $\mathrm{Tb}<\mathrm{T}_{\text {méd }} \leq \mathrm{Tot} \mathrm{e}$ $\mathrm{STd}=\left\{(\mathrm{Tot}-\mathrm{Tb}) .\left[\left(\mathrm{TB}-\mathrm{T}_{\text {méd }}\right) /(\mathrm{TB}-\mathrm{Tot})\right]\right\} .1$ dia, quando Tot $<\mathrm{T}_{\text {méd }} \leq \mathrm{TB}$; se $\mathrm{T}_{\text {méd }}<\mathrm{Tb}$, então $\mathrm{T}_{\text {méd }}=\mathrm{Tb}$; se $\mathrm{T}_{\text {méd }}>\mathrm{TB}$, então $\mathrm{T}_{\text {méd }}=\mathrm{TB}$ Método 3.2: $\mathrm{STd}=\left(\mathrm{T}_{\text {méd }}-\mathrm{Tb}\right) .1$ dia, quando $\mathrm{Tb}<\mathrm{T}_{\text {méd }} \leq \mathrm{Tot} \mathrm{e}$ $\mathrm{STd}=\left\{(\mathrm{Tot}-\mathrm{Tb}) \cdot\left[\left(\mathrm{TB}-\mathrm{T}_{\text {méd }}\right) /(\mathrm{TB}-\mathrm{Tot})\right]\right\} .1 \mathrm{dia}$, quando Tot $<T_{\text {méd }}<T B$; se $T_{\text {mín }}<T b$, então $T_{\text {mín }}=T b$; se $T_{\text {máx }}>T B$, então $T_{\text {máx }}=T B$, em que Tmed é a temperatura média diária do ar, calculada pela média aritmética entre a temperatura mínima $\left(\mathrm{T}_{\text {min }}\right)$ e a temperatura máxima $\left(\mathrm{T}_{\text {máx }}\right)$ diária do ar; e Tb, Tot e TB são as temperaturas cardinais de aparecimento de folhas (temperatura base inferior, temperatura ótima e temperatura base superior, respectivamente). Considerou-se $\mathrm{Tb}=0^{\circ} \mathrm{C}$, Tot $=22^{\circ} \mathrm{C}$ e $\mathrm{TB}=35^{\circ} \mathrm{C}$ (Streck et al., 2003b).

A soma térmica diária foi acumulada a partir da emergência e resultou na soma térmica acumulada $\left(\mathrm{STa},{ }^{\circ} \mathrm{C}\right.$ dia $)$, calculada por STa $=\sum \mathrm{STd}$. Para cada método de cálculo da soma térmica (equações 2 a 7), foi realizada uma regressão linear simples entre HS no colmo principal e STa para cada repetição (média das seis plantas por parcela) (Streck et al., 2005a). 
O filocrono foi estimado como sendo o inverso do coeficiente angular (declividade) dessa regressão linear (Klepper et al., 1982; Xue et al., 2004). Para cada método de cálculo da soma térmica, foi calculado o desvio-padrão (DP) da variável filocrono, entre as 13 datas de semeadura, para cada cultivar. O DP foi usado para selecionar o método de cálculo da soma térmica para determinação do filocrono, já que essa estatística descritiva é uma medida de dispersão da variável. Selecionado-se o melhor método de cálculo da soma térmica, as médias de filocrono foram submetidas à análise de variância para avaliar o efeito das fontes de variação (cultivares e datas de semeadura) e comparadas pelo teste de Scott-Knott, a 5\% de probabilidade.

\section{Resultados e Discussão}

Nos três anos de condução do experimento, durante o período de emissão de folhas, as temperaturas mínima e máxima absolutas observadas oscilaram entre $-1,7^{\circ} \mathrm{C}$ $(29 / 7 / 2007)$ e $38,6^{\circ} \mathrm{C}(8 / 1 / 2006)$, respectivamente. Durante os meses de junho a agosto, foram frequentes os dias com temperatura mínima do ar menor que a Tot $\left(22^{\circ} \mathrm{C}\right)$ e próxima da $\mathrm{Tb}\left(0^{\circ} \mathrm{C}\right)$. Nos meses de novembro a março, foram frequentes os dias em que a temperatura máxima do ar foi maior que a Tot e próxima da TB $\left(35^{\circ} \mathrm{C}\right)$. As plantas de trigo também foram expostas à variação máxima de fotoperíodo nesse local, entre 11,1 e 15,0 horas, o que ocorre respectivamente entre o solstício de inverno (21/6) e o solstício de verão (21/12). Essas condições distintas de ambiente são importantes em estudos ecofisiológicos para a caracterização dos parâmetros do desenvolvimento da cultura do trigo, que, em razão das baixas temperaturas cardinais, tem seu desenvolvimento fortemente modificado durante os meses de verão (Streck et al., 2003b).
Elevados valores do coeficiente de determinação $\left(\mathrm{R}^{2}\right.$ acima de 0,95$)$ entre HS e STa foram obtidos para todas as cultivares, para todas as datas de semeadura e em todos o métodos de cálculo da soma térmica, o que indica que a estimativa do filocrono pelo método da regressão linear entre HS e STa é apropriada (Xue et al., 2004; Streck et al., 2005a,b). Nas seis cultivares, o filocrono foi maior com os métodos 1.1 e 1.2 , intermediário com os métodos 2.1 e 2.2 , e menor com os métodos 3.1 e 3.2 , com variação entre $84,0^{\circ} \mathrm{C}$ e $140,1^{\circ} \mathrm{C}$ dia folha ${ }^{-1}$ (Tabela 1 ). A redução do filocrono, quando se usou a Tot (métodos 2.1 e 2.2) ou a Tot e a TB (métodos 3.1 e 3.2), ocorreu em razão dos menores valores de STd, calculados por esses métodos, nas semeaduras realizadas nos períodos mais quentes do ano, quando a temperatura média do ar ficou acima da Tot $\left(22^{\circ} \mathrm{C}\right)$ em muitos dias. Nesses dias, a STd é, no máximo, igual à Tot (métodos 2.1 e 2.2), ou há uma redução gradativa da $\mathrm{STd}$ até a TB (métodos $3.1 \mathrm{e}$ 3.2). Já entre os métodos 1.1 e 1.2, a STd foi similar, pois a $T_{\text {méd }}$ não foi inferior à $T b$ e a $T_{\text {mín }}$ foi inferior à $\mathrm{Tb}$ em apenas três dias, o que explica os valores iguais de filocrono com esses dois métodos para todas as cultivares (Tabela 1). Os valores de filocrono determinados com o método 1.1 variaram de 116,2 a $140,1^{\circ} \mathrm{C}$ dia folha ${ }^{-1}$, variação menor do que a de 57,2 a $116,2^{\circ} \mathrm{C}$ dia folha ${ }^{-1}$ relatada em Cao \& Moss (1989), para cultivares europeias de trigo, e mais próxima da faixa de 92 a $147^{\circ} \mathrm{C}$ dia folha ${ }^{-1}$ relatada por Streck et al. (2005b), para cultivares brasileiras de trigo, usando o método 1.1.

O desvio-padrão (DP) do filocrono com a soma térmica calculada pelos seis métodos (equações 2 a 7) variou de 4,15 a $8,36^{\circ} \mathrm{C}$ dia folha- ${ }^{-1}$ (Tabela 1 ). $\mathrm{O}$ DP diminuiu à medida que se incorporaram no cálculo a Tot e a TB, tanto quando se compararam as temperaturas cardinais ( $\mathrm{Tb}$, Tot e TB) com a Tmed

Tabela 1. Média \pm desvio-padrão do filocrono $\left({ }^{\circ} \mathrm{C}\right.$ dia folha $\left.{ }^{-1}\right)$ de seis cultivares de trigo em 13 datas de semeadura com a soma térmica calculada por seis métodos.

\begin{tabular}{|c|c|c|c|c|c|c|}
\hline \multirow[t]{2}{*}{ Cultivar } & \multicolumn{6}{|c|}{ Métodos de cálculo da soma térmica } \\
\hline & $\begin{array}{l}\text { Método 1.1 } \\
\text { (equação 2) }\end{array}$ & $\begin{array}{l}\text { Método } 1.2 \\
\text { (equação 3) }\end{array}$ & $\begin{array}{l}\text { Método } 2.1 \\
\text { (equação 4) }\end{array}$ & $\begin{array}{l}\text { Método } 2.2 \\
\text { (equação 5) }\end{array}$ & $\begin{array}{l}\text { Método } 3.1 \\
\text { (equação 6) }\end{array}$ & $\begin{array}{l}\text { Método } 3.2 \\
\text { (equação } 7 \text { ) }\end{array}$ \\
\hline BRS Louro & $116,2 \pm 6,95$ & $116,2 \pm 6,92$ & $109,7 \pm 6,22$ & $104,1 \pm 5,89$ & $99,7 \pm 6,30$ & $84,0 \pm 4,90$ \\
\hline CEP 51 & $120,5 \pm 7,08$ & $120,5 \pm 7,06$ & $114,1 \pm 6,66$ & $108,1 \pm 6,26$ & $104,4 \pm 6,46$ & $88,0 \pm 5,37$ \\
\hline CEP 52 & $124,5 \pm 5,43$ & $124,5 \pm 5,48$ & $117,8 \pm 5,02$ & $112,0 \pm 4,76$ & $107,4 \pm 5,38$ & $90,9 \pm 4,15$ \\
\hline BRS 177 & $134,9 \pm 8,31$ & $134,9 \pm 8,36$ & $128,4 \pm 7,87$ & $121,6 \pm 7,34$ & $117,2 \pm 6,87$ & $99,3 \pm 5,81$ \\
\hline Nova Era & $124,3 \pm 5,76$ & $124,3 \pm 5,76$ & $118,0 \pm 5,73$ & $111,6 \pm 5,50$ & $107,8 \pm 5,33$ & $91,0 \pm 4,33$ \\
\hline BRS Tarumã & $140,1 \pm 7,14$ & $140,1 \pm 7,13$ & $131,9 \pm 7,14$ & $123,0 \pm 6,03$ & $119,5 \pm 5,73$ & $100,2 \pm 6,08$ \\
\hline Média & $126,8 \pm 6,78$ & $126,8 \pm 6,79$ & $120,0 \pm 6,44$ & $113,4 \pm 5,96$ & $109,3 \pm 6,01$ & $92,2 \pm 5,11$ \\
\hline
\end{tabular}


(DP pelo método 1.1 >método $2.1>$ método 3.1) como quando se compararam as temperaturas cardinais ( $\mathrm{Tb}$, Tot e TB) com a $\mathrm{T}_{\text {mín }}$ e $\mathrm{T}_{\text {máx }}$ (DP pelo método $1.2>$ método $2.2>$ método 3.2). Na comparação da temperatura do ar com as temperaturas cardinais, $o$ DP diminuiu quando Tb, Tot e TB foram comparadas com a $\mathrm{T}_{\text {mín }}$ e $\mathrm{T}_{\text {máx }}$ (métodos 2.2 e 3.2 ), com o menor DP pelo método 3.2 para todas as cultivares. No método 3.2, considera-se a resposta da emissão de folhas às três temperaturas cardinais, e a comparação das temperaturas cardinais é feita com as temperaturas mínima e máxima do ar. Esse deve ser, portanto, o método a ser utilizado para calcular o filocrono em trigo, pois a emissão de folhas é resultado de processos que envolvem diferenciação dos primórdios foliares, divisão e crescimento celular. Esses processos são desencadeados por reações enzimáticas que, por sua natureza proteica, desnaturam em temperaturas elevadas, e a velocidade de catalização das reações químicas é reduzida quando a temperatura é superior a uma temperatura ótima (Streck, 2004). Para a cultura da soja, o uso das temperaturas mínima e máxima diárias do ar também melhorou a simulação do desenvolvimento vegetativo em relação ao uso da temperatura média diária do ar (Streck et al., 2009). O fato de a comparação das temperaturas cardinais com as a $\mathrm{T}_{\text {mín }}$ e a $\mathrm{T}_{\text {máx }}$, e não com a $\mathrm{T}_{\text {méd, }}$ ter apresentado melhor desempenho entre os métodos indica que a emissão de folhas em trigo deve ser descrita em modelos matemáticos em resposta às temperaturas extremas do dia e não à temperatura média. Assim, o filocrono calculado com a soma térmica pelo método 3.2 (equação 7) foi a variável usada nas análises posteriores.

A análise de variância para a variável filocrono com a soma térmica calculada pelo método 3.2 mostrou efeito significativo a $5 \%$ para os fatores principais (cultivares e datas de semeadura) e a interação dos fatores. Assim, a análise estatística foi desdobrada dentro de cada fator (Tabela 2 e Figura 1). O filocrono entre as cultivares diferiu dependendo da data de semeadura e, na média das 13 datas de semeadura, o filocrono teve a sequência BRS Tarumã $>$ BRS177 > Nova Era >CEP $52>$ CEP $51>$ BRS Louro (Tabela 2), ou seja, as cultivares que apresentaram maiores valores médios de filocrono foram as de ciclo tardio (BRS Tarumã e Nova Era) e médio (BRS 177). Cultivares mais tardias têm maior número final de folhas (NFF) no colmo principal e, assim, o maior filocrono nessas cultivares pode ser explicado, segundo Skinner \& Nelson (1995), pelo maior filocrono das últimas folhas, já que a velocidade de surgimento de folhas diminui à medida que novas folhas vão aparecendo no colmo, em função da maior distância entre o ápice meristemático e a extremidade do cartucho (Streck et al., 2003b). Observou-se que em 3 das 13 datas de semeadura (24/6/2005, 7/9/2005 e 21/12/2005) não houve diferença entre cultivares. Nas outras dez datas de semeadura, as diferenças de filocrono entre cultivares foram variáveis (Tabela 2) e apresentaram desde diferenças significativas apenas entre a cultivar BRS Tarumã (maior filocrono) e as demais, que não diferiram entre si (datas de semeadura 26/10/2005 e 4/9/2006), até três grupos de diferenças

Tabela 2. Médias do filocrono $\left({ }^{\circ} \mathrm{C}\right.$ dia folha ${ }^{-1}$ ) calculado com a soma térmica pelo método 3.2 (equação 7) de seis cultivares de trigo em 13 datas de semeadura ${ }^{(1)}$.

\begin{tabular}{|c|c|c|c|c|c|c|}
\hline Datas de semeadura & BRS Tarumã & BRS 177 & Nova Era & CEP 51 & CEP 52 & BRS Louro \\
\hline $24 / 6 / 2005$ & $91,0 \mathrm{a}$ & $94,3 \mathrm{a}$ & $86,2 \mathrm{a}$ & $85,1 \mathrm{a}$ & $82,9 \mathrm{a}$ & $78,2 \mathrm{a}$ \\
\hline $4 / 8 / 2005$ & $110,6 \mathrm{a}$ & $101,4 a$ & $93,6 \mathrm{~b}$ & $94,9 \mathrm{~b}$ & $87,0 \mathrm{~b}$ & $90,5 \mathrm{~b}$ \\
\hline 7/9/2005 & $93,8 \mathrm{a}$ & $95,5 \mathrm{a}$ & $89,5 \mathrm{a}$ & $88,5 \mathrm{a}$ & $82,7 \mathrm{a}$ & $89,3 \mathrm{a}$ \\
\hline $26 / 10 / 2005$ & $75,6 \mathrm{a}$ & $59,8 \mathrm{~b}$ & $72,5 \mathrm{a}$ & $62,0 \mathrm{~b}$ & $62,6 \mathrm{~b}$ & $60,1 \mathrm{~b}$ \\
\hline $21 / 12 / 2005$ & $86,1 \mathrm{a}$ & $92,1 \mathrm{a}$ & $86,2 \mathrm{a}$ & $83,7 \mathrm{a}$ & $88,6 \mathrm{a}$ & $78,5 \mathrm{a}$ \\
\hline $6 / 2 / 2006$ & $113,6 a$ & $91,4 b$ & $84,0 \mathrm{~b}$ & $81,9 b$ & $87,7 \mathrm{~b}$ & $64,9 \mathrm{c}$ \\
\hline $21 / 3 / 2006$ & $120,7 \mathrm{a}$ & $127,9 \mathrm{a}$ & $108,8 b$ & $99,0 \mathrm{~b}$ & $98,8 \mathrm{~b}$ & $87,0 \mathrm{c}$ \\
\hline $12 / 5 / 2006$ & $119,4 b$ & $132,5 \mathrm{a}$ & $112,8 \mathrm{~b}$ & $107,9 \mathrm{~b}$ & $104,6 b$ & $111,6 \mathrm{~b}$ \\
\hline $7 / 7 / 2006$ & ${ }^{(2)}$ & $100,7 \mathrm{a}$ & $90,9 b$ & $84,8 \mathrm{~b}$ & $85,2 \mathrm{~b}$ & $84,5 b$ \\
\hline 4/9/2006 & $99,8 \mathrm{a}$ & $86,8 \mathrm{~b}$ & $76,5 b$ & $79,0 \mathrm{~b}$ & $79,8 \mathrm{~b}$ & $73,8 b$ \\
\hline $25 / 10 / 2006$ & $74,3 \mathrm{c}$ & $70,7 \mathrm{c}$ & $67,4 b$ & $68,9 \mathrm{~b}$ & $67,7 \mathrm{~b}$ & $81,0 \mathrm{a}$ \\
\hline $18 / 4 / 2007$ & $125,4 a$ & $125,2 \mathrm{a}$ & $112,3 b$ & $104,1 b$ & $108,4 b$ & $97,1 \mathrm{~b}$ \\
\hline $7 / 6 / 2007$ & $94,1 \mathrm{~b}$ & $113,4 \mathrm{a}$ & $101,7 \mathrm{~b}$ & $78,6 \mathrm{~b}$ & $101,0 \mathrm{~b}$ & $94,1 \mathrm{~b}$ \\
\hline Média & 100,4 & 99,4 & 91,0 & 86,0 & 87,5 & 83,9 \\
\hline
\end{tabular}

${ }^{(1)}$ Médias com letras iguais na linha não diferem entre si pelo teste de Scott-Knott, a 5\% de probabilidade. (2)Perda de todas as parcelas da cultivar. O coeficiente de variação da análise da variância foi de $9,0 \%$. 
significativas (datas de semeadura 6/2/2006, 21/3/2006 e 25/10/2006). Em todas as datas de semeadura, o menor filocrono foi da cultivar BRS Louro, que diferiu do das cultivares CEP 52 e CEP 51 apenas nas semeaduras de 6/2/2006 e 21/3/2006. A cultivar BRS Louro teve o maior filocrono e diferiu das outras cinco cultivares em apenas uma data de semeadura (25/10/2006). Nessa data de semeadura, BRS Louro teve o menor NFF (média geral de oito folhas) e teve apenas três valores de HS (usados na regressão entre o HS e a STa), o que pode ter levado a algum erro na estimativa do filocrono e, portanto, explicar o maior filocrono dessa cultivar. $\mathrm{O}$ efeito do genótipo no filocrono em trigo tem sido um assunto controverso na literatura. Klepper et al. (1982) e McMaster et al. (1992) não encontraram diferenças de filocrono entre várias cultivares de trigo de inverno, enquanto Cao \& Moss (1989), Frank \& Bauer (1995) e Streck et al. (2005b) relataram diferenças de filocrono
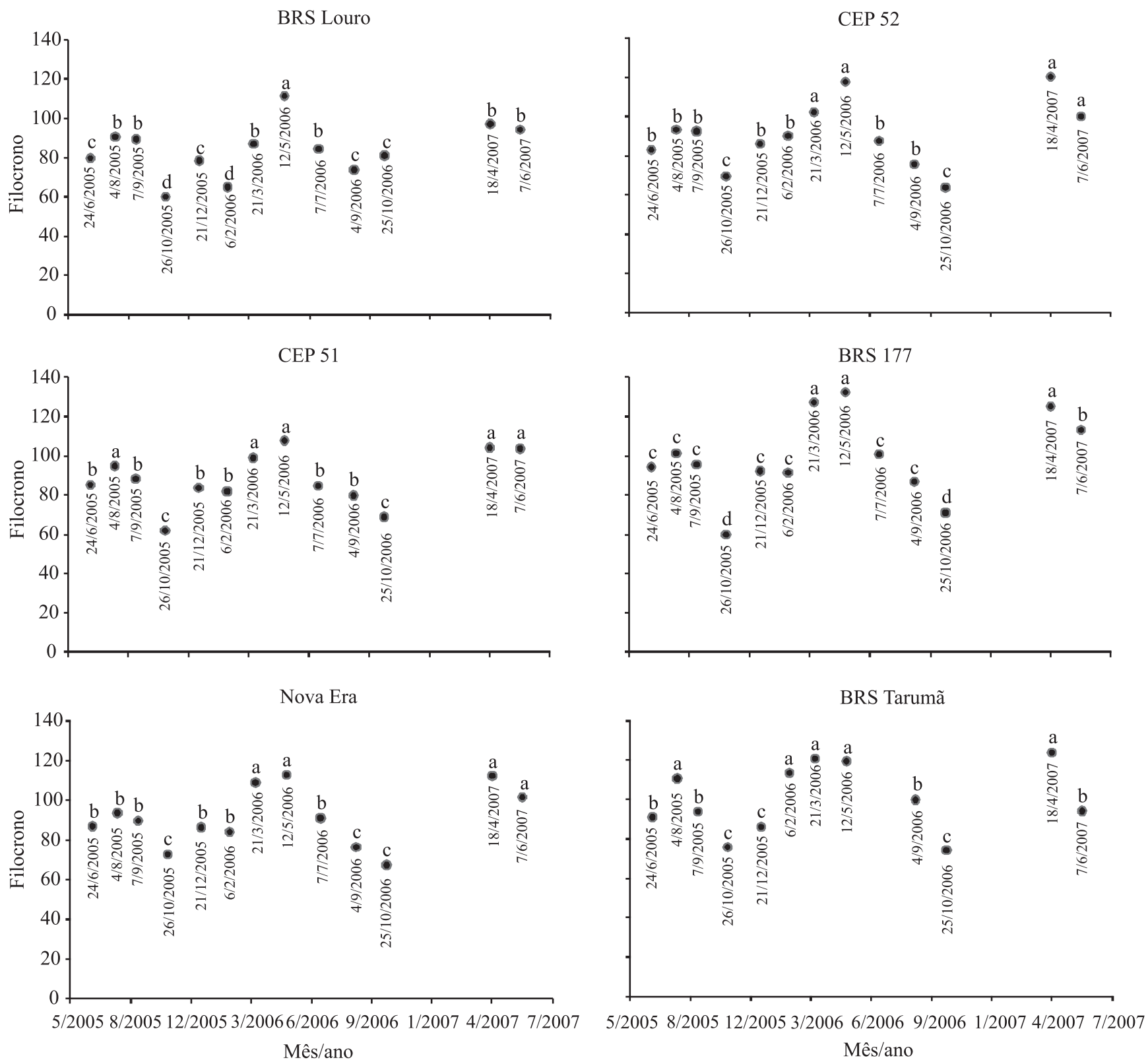

Figura 1. Média do filocrono $\left({ }^{\circ} \mathrm{C}\right.$ dia folha $\left.{ }^{-1}\right)$ calculado com a soma térmica pelo método 3.2 (equação 7 ) de seis cultivares de trigo em 13 datas de semeadura. Médias com letras diferentes em cada painel (cultivar) indicam diferenças entre datas de semeadura (indicadas abaixo de cada ponto) pelo teste de Scott-Knott, a 5\% de probabilidade. 
entre cultivares de trigo. Os resultados deste trabalho (Tabela 2) concordam com os que foram obtidos pelos últimos autores.

O filocrono variou entre as datas de semeadura, com menores valores nas semeaduras durante a primavera e o verão e maiores valores nas semeaduras durante o outono e o inverno (Figura 1), o que concorda com os resultados de Jamieson et al. (2008). Essa variação ao longo do ano sugere uma resposta do filocrono ao fotoperíodo. Na Figura 2, está plotado o filocrono em relação ao fotoperíodo médio durante o período de emissão de folhas, para as seis cultivares. Nota-se, em todas as cultivares, uma relação linear decrescente do filocrono com o aumento no fotoperíodo, com $\mathrm{R}^{2}$ entre
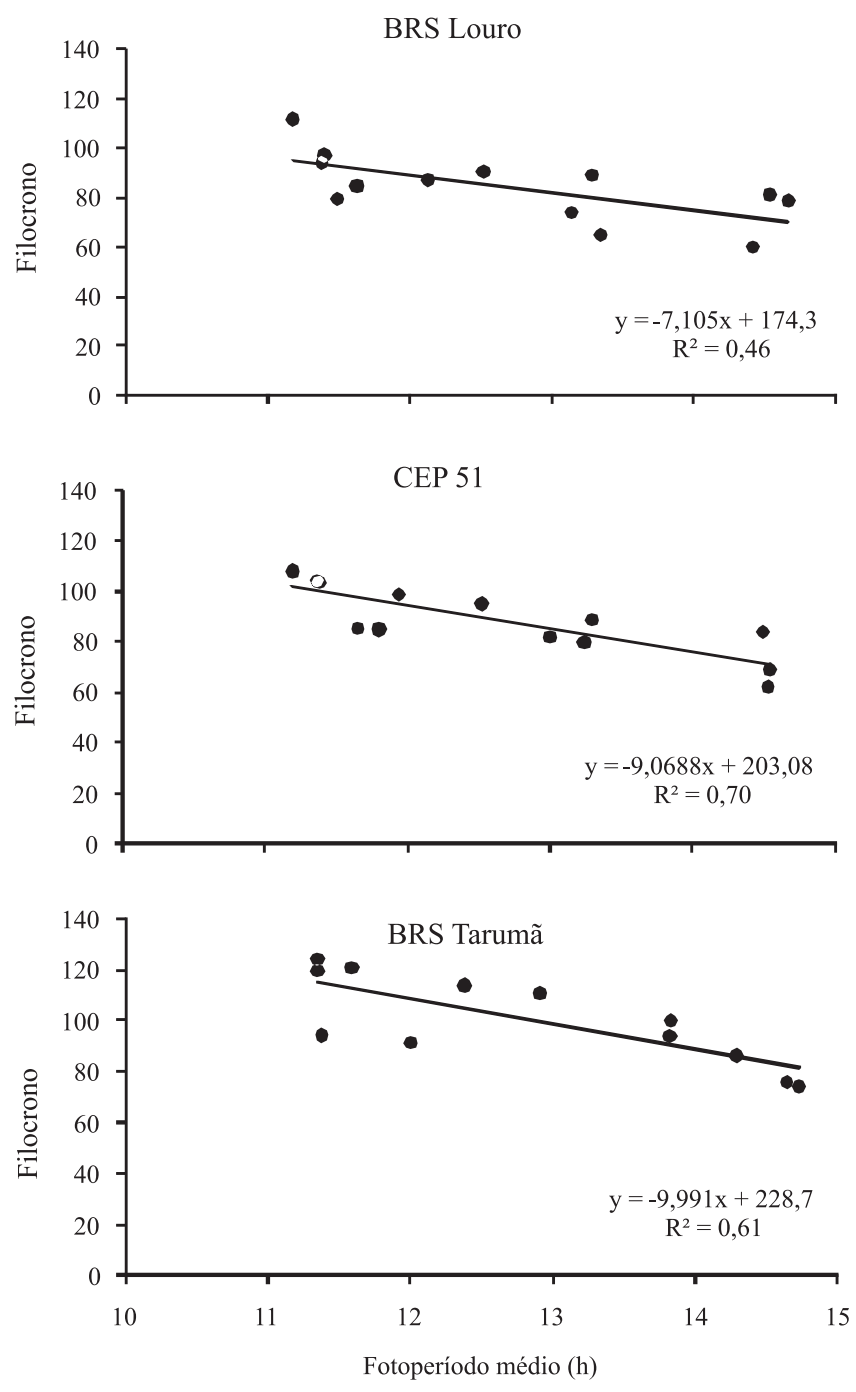

0,45 e 0,72 e o coeficiente angular significativo a $5 \%$ de probabilidade para todas as cultivares. A relação decrescente na Figura 2 indica que o desenvolvimento (velocidade de emissão de folhas) aumenta com o aumento do fotoperíodo, uma resposta típica de plantas de dia longo, o que concorda com relatos anteriores sobre trigos de inverno (Cao \& Moss, 1989; Streck et al., 2003b; Bassu et al., 2009). Na Figura 2, também se observa que a resposta fotoperiódica é distinta entre as cultivares. O coeficiente angular das equações apresentadas na Figura 2 indica a sensibilidade ao fotoperíodo, pois representa o decréscimo do filocrono para cada hora de aumento no fotoperíodo. O coeficiente angular foi de 7,1 para $14,2\left({ }^{\circ} \mathrm{C}\right.$ dia folha $\left.{ }^{-1}\right) \mathrm{h}^{-1}$ da
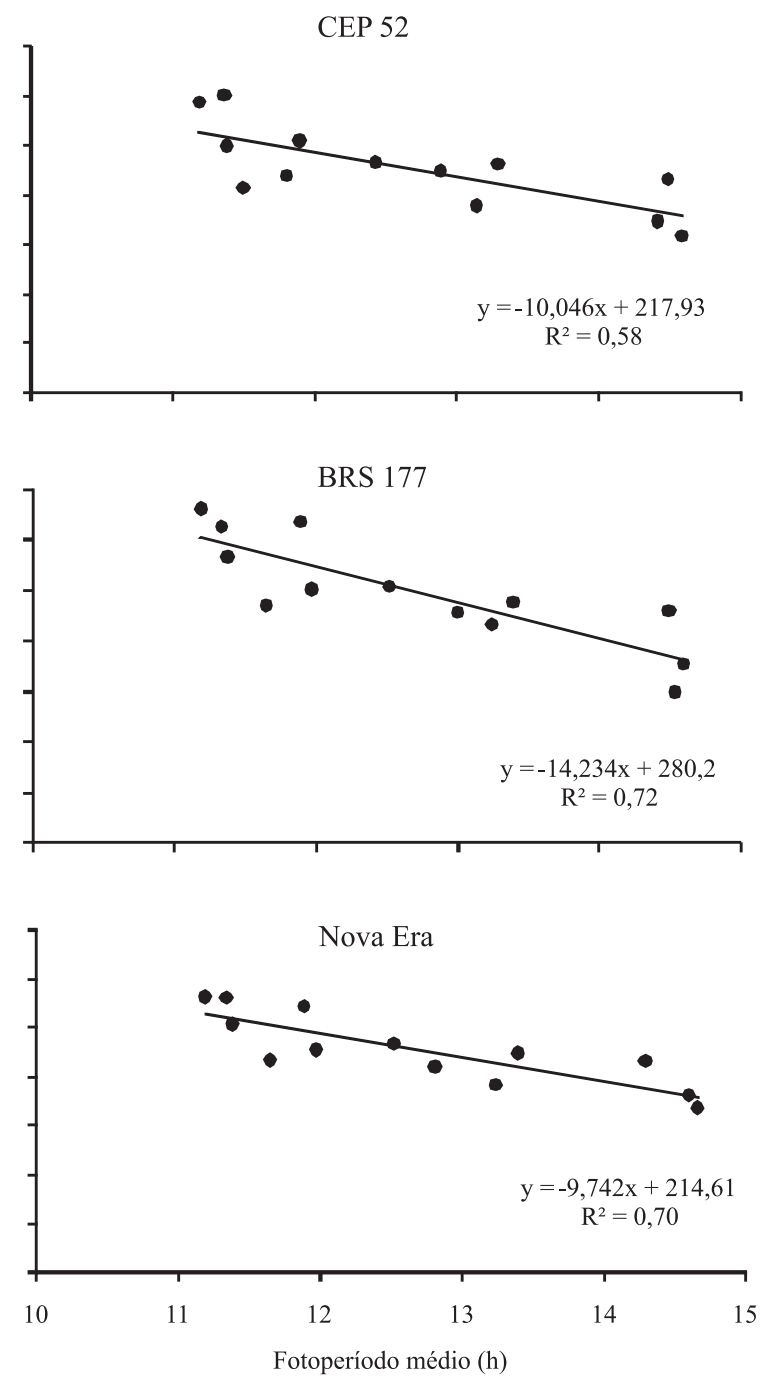

Figura 2. Relação entre filocrono $\left({ }^{\circ} \mathrm{C}\right.$ dia folha $\left.{ }^{-1}\right)$ e fotoperíodo médio (horas) durante o período de emissão de folhas para 6 cultivares de trigo em 13 datas de semeadura, em Santa Maria, RS, de 2005 a 2007. 
cultivar menos sensível para a cultivar mais sensível, e foi menor nas duas cultivares precoces (BRS Louro e CEP 51) e maior na cultivar média BRS 177. As duas cultivares tardias (BRS Tarumã e Nova Era) e a cultivar média CEP 52 ficaram na posição intermediária. Esses resultados indicam que a sensibilidade do trigo ao fotoperíodo está associada ao grupo de maturação da cultivar, de modo que, em geral, quanto mais precoce for a cultivar, menor é a sensibilidade ao fotoperíodo durante a emissão de folhas, o que concorda com resultados encontrados para trigos de inverno (Streck et al., 2003a,b).

O filocrono tem sido uma variável do desenvolvimento vegetativo bastante usada em estudos ecofisiológicos e em modelos de simulação do crescimento, desenvolvimento e produtividade de trigo (McMaster et al., 1991; Streck et al., 2003b; Bassu et al., 2009). É uma variável atraente por sua relativa facilidade de obtenção (medida ou estimada) e por estar associada a outros eventos relacionados ao crescimento e desenvolvimento, como a área foliar e o início do desenvolvimento reprodutivo (McMaster et al., 1991; Streck et al., 2003a,b). No entanto, os resultados deste estudo se somam a resultados anteriores que dizem haver variação entre cultivares e entre datas de semeadura. Os resultados deste trabalho, no entanto, indicam o método de cálculo da soma térmica como um dos fatores adicionais que afetam a estimativa do filocrono de trigo em diferentes épocas de semeadura, o que ainda não havia sido descrito na literatura. Apesar da sua dependência de vários fatores, o filocrono foi usado recentemente por Bassu et al. (2009) em um modelo de simulação da produtividade em trigo que considera a variação do filocrono em relação à época de semeadura de forma simples (fotoperíodo na espigueta terminal), o que não comprometeu a aplicação prática do modelo matemático, indicando que, apesar de sua sensibilidade a diversos fatores, é vantajoso usar o filocrono como variável em modelos de simulação em trigo.

\section{Conclusões}

1. A estimativa do filocrono em trigo é afetada pelo método de cálculo da soma térmica.

2. O método que utiliza as três temperaturas cardinais de emissão de folhas e a comparação das temperaturas cardinais com a temperatura mínima e máxima do ar melhora a precisão na simulação do desenvolvimento vegetativo dessa cultura, em comparação aos demais métodos

3. O filocrono varia com a cultivar de trigo, sendo que as cultivares mais precoces têm menor filocrono do que cultivares tardias.

4. Ofilocronoemtrigo varia comaépocade semeadura em resposta ao fotoperíodo e à temperatura.

\section{Agradecimentos}

À Fundação de Amparo à Pesquisa do Estado do Rio Grande do Sul, à Coordenação de Aperfeiçoamento de Pessoal de Nível Superior e ao Conselho Nacional de Desenvolvimento Científico e Tecnológico, pelas bolsas de iniciação científica, mestrado, doutorado e produtividade em pesquisa concedidas.

\section{Referências}

BASSU, S.; ASSENG, S.; MOTZO, R.; GIUNTA, F. Optimising sowing date of durum wheat in a variable Mediterranean environment. Field Crops Research, v.111, p.109-118, 2009.

CAO, W.X.; MOSS, D.N. Temperature and daylength interaction on phyllochron in wheat and barley. Crop Science, v.29, p.1046-1048, 1989.

DELlAI, J.; TRENTIN, G.; BISOGNIN, D.A.; STRECK, N.A. Filocrono em diferentes densidades de plantas de batata. Ciência Rural, v.35, p.1269-1274, 2005.

FRANK, A.B.; BAUER, A. Phyllochron differences in wheat, barley, and forage grasses. Crop Science, v.35, p.19-23, 1995.

GILMORE JUNIOR, E.C.; ROGERS, J.S. Heat units as a method of measuring maturity in corn. Agronomy Journal, v.50, p.611-615, 1958.

HERMES, C.C.; MEDEIROS, S.L.P.; MANFRON, P.A.; CARON, B.; POMMER, S.F.; BIANCHI, C. Emissão de folhas de alface em função da soma térmica. Revista Brasileira de Agrometeorologia, v.9, p.269-275, 2001.

JAMIESON, P.; BROOKING, I.; ZYSKOWSKI, R.; MUNRO, $\mathrm{C}$. The vexatious problem of the variation of the phyllochron in wheat. Field Crops Research, v.108, p.163-168, 2008.

KLEPPER, B.; RICKMAN, R.W.; PETERSON, C.M. Quantitative characterization of vegetative development in small cereal grains. Agronomy Journal, v.74, p.789-792, 1982.

MCMASTER, G.S. Phytomers, phyllochrons, phenology and temperate cereal development. Journal of Agricultural Science, v.143, p.137-150, 2005.

MCMASTER, G.S.; KLEPPER, B.; RICKMAN, R.W.; WILHELM, W.W.; WILLIS, W.O. Simulation of shoot vegetative development and growth of unstressed winter wheat. Ecological Modeling, v.53, p.189-204, 1991. 
MCMASTER, G.S.; WILHELM, W.W. Growing degree-days: one equation, two interpretations. Agricultural and Forest Meteorology, v.87, p.291-300, 1997.

MCMASTER, G.S.; WILHELM, W.W.; MORGAN, J.A. Simulating winter wheat shoot apex phenology. Journal of Agricultural Science, v.119, p.1-12, 1992.

PAULA, F.L.M.; STRECK, N.A.; BISOGNIN, D.A.; HELDWEIN, A.B.; LAGO, I. Filocrono da planta de batata cultivar Asterix em diferentes épocas de plantio. Revista Brasileira de Agrometeorologia, v.13, p.367-374, 2005.

REUNIÃO DA COMISSÃO SUL-BRASILEIRA DE PESQUISA DE TRIGO E TRITICALE, 38; REUNIÃO DA COMISSÃO CENTRO-SUL BRASILEIRA DE PESQUISA DE TRIGO E TRITICALE, 21., 2006, Passo Fundo. Informações técnicas para a safra 2007: trigo e triticale. Passo Fundo: Embrapa Trigo, 2006. 114p. (Embrapa Trigo. Documentos, 71).

SINCLAIR, T.R.; GILBERT, R.A.; PERDOMO, R.E.; SHINE JUNIOR, J.M.; POWELL, G.; MONTES, G. Sugarcane leaf area development under field conditions in Florida, USA. Field Crops Research, v.88, p.171-178, 2004.

SKINNER, R.H.; NELSON, C.J. Elongation of the grass leaf and its relationship to the phyllochron. Crop Science, v.35, p.4-10, 1995.

SOCIEDADE BRASILEIRA DE CIÊNCIA DO SOLO. Manual de adubação e de calagem para os Estados do Rio Grande do Sul e Santa Catarina. 10.ed. Porto Alegre: Sociedade Brasileira de Ciência do Solo, 2004. 400p.

STRECK, N.A. A temperature response function for modeling leaf growth and development of the African violet (Saintpaulia ionantha Wendl.). Ciência Rural, v.34, p.55-62, 2004.

STRECK, N.A.; BELLÉ, R.A.; ROCHA, E.K. da; SCHUH, M. Estimating leaf appearance and phyllochron in safflower (Carthamus tinctorius L.). Ciência Rural, v.35, p.1448-1450, 2005a.

STRECK, N.A.; BOSCO, L.C.; MENEZES, N.L.; GARCIA, D.C.; ALBERTO, C.M.; LAGO, I. Estimativa do filocrono em cultivares de trigo de primavera. Revista Brasileira de Agrometeorologia, v.13, p.423-429, 2005 b.

STRECK, N.A.; BOSCO, L.C.; MICHELON, S.; WALTER, L.C.; MARCOLIN, E. Duração do ciclo de desenvolvimento de arroz em função da emissão de folhas no colmo principal. Ciência Rural, v.36, p.1086-1093, 2006.

STRECK, N.A.; MICHELON, S.; ROSA, H.T.; WALTER, L.C.; BOSCO, L.C.; PAULA, G.M. de; CAMERA, C.; SAMBORANHA, F.K.; MARCOLIN, E.; LOPES, S.J. Filocrono de genótipos de arroz irrigado em função da época de semeadura. Ciência Rural, v.37, p.323-329, 2007a.

STRECK, N.A.; PAULA, F.L.M. de; BISOGNIN, D.A.; HELDWEIN, A.B.; DELLAI, J. Simulating the development of field grown potato (Solanum tuberosum L.). Agricultural and Forest Meteorology, v.142, p.1-11, 2007b.

STRECK, N.A.; PAULA, G.M. de; OLIVEIRA, F.O.; SCHWANTES, A.P.; MENEZES, N.L. de. Improving node number simulation in soybean. Pesquisa Agropecuária Brasileira, v.44, p.661-668, 2009.

STRECK, N.A.; WEISS, A.; XUE, Q.; BAENZIGER, P.S. Improving predictions of developmental stages in winter wheat: a modified Wang and Engel model. Agricultural and Forest Meteorology, v.115, p.139-150, 2003a.

STRECK, N.A.; WEISS, A.; XUE, Q.; BAENZIGER, P.S. Incorporating a chronology response into the prediction of leaf appearance rate in winter wheat. Annals of Botany, v.92, p.181-190, 2003b.

UNITED STATES DEPARTMENT OF AGRICULTURE. Production, supply and distribution online: custom query. Disponível em: <http://www.fas.usda.gov/psdonline/psdQuery. aspx>. Acesso em: 15 jan. 2009.

WILHELM, W.W.; MCMASTER, G.S. Importance of the phyllochron in studying development and growth in grasses. Crop Science, v.35, p.1-3, 1995.

XUE, Q.W.; WEISS, A.; BAENZIGER, P.S. Predicting leaf appearance in field-grown winter wheat: evaluating linear and non-linear models. Ecological Modelling, v.175, p.261-270, 2004.

Recebido em 6 de março de 2009 e aprovado em 26 de outubro de 2009 